\title{
Congenital Glioblastoma: Lessons Learned from a Rare Case with Unusual Presentation
}

\author{
Atef BEN NSIR ${ }^{1}$, Mehdi DARMOUL ${ }^{1}$, Rym HADHRI ${ }^{2}$, Nejib HATTAB ${ }^{1}$ \\ ${ }^{1}$ Fattouma Bourguiba University Hospital, Department of Neurosurgery, Monastir, Tunisia \\ ${ }^{2}$ Fattouma Bourguiba University Hospital, Department of Pathology, Monastir, Tunisia
}

\section{ABSTRACT}

Congenital glioblastomas are uncommon tumors of infancy with unique molecular features and usually better prognosis compared to their pediatric and adult counterparts. A 15-day old newborn harboring a congenital glioblastoma undiagnosed at pregnancy presented to our emergencies with rapid neurological deterioration. Radiological investigations revealed an 8 by $9 \mathrm{~cm}$ space occupying and enhancing lesion in the left temporoparietooccipital region with intratumoral hemorrhage. The patient was immediately transferred to the operative theater and was operated in a poor condition. Unfortunately he showed no improvement post-operatively and died within two days in the intensive care unit. As such presentation is unique; we present the case and review the relevant literature concerning the potential pitfalls in diagnosis, management strategies and improvements in outcome.

KEYWORDS: Congenital glioblastoma, Magnetic resonance imaging, Surgery, Chemotherapy, Prognosis

ABBREVIATIONS: CNS: Central nervous system, CT: Computed tomography, MRI: Magnetic resonance imaging, EGFR: Epidermal growth factor receptor, GFAP: Glial fibrillary acidic protein

\section{INTRODUCTION}

$\longrightarrow$ ongenital brain tumors account for approximately 0.3 to $4 \%$ of all central nervous system (CNS) tumors in infancy (4). Glioblastoma (GBM) subtype is extremely rare, representing only $3.5 \%$ of all these cases with less than 50 cases reported in the English medical literature.

Due to its highly aggressive behavior; its prognosis was regarded as very poor for a long time. But recent evidence suggests a better outcome of these congenital forms compared to their pediatric and adult counterparts (3).

We report an exceptional case of congenital glioblastoma undiagnosed at pregnancy who presented in emergency with rapid neurological deterioration and review the relevant literature concerning the potential pitfalls in diagnosis, management strategies and improvements in outcome.

\section{CASE REPORT}

A 24-year-old primigravida gave birth to a male baby after a supposed normal pregnancy. Apgar scores were 9 and 10 at 1 and 5 minutes post-delivery, respectively. The birth weight was $3.5 \mathrm{~kg}$, height $49 \mathrm{~cm}$ and head circumference was $36 \mathrm{~cm}$. The anterior fontanel was not tense and the cranial sutures were normal. The newborn's pupils were isocoric and he had positive pupillary light reflexes. He also exhibited normal startle, rooting and sucking reflexes. There was no evidence of motor weakness.

On day 15 , he presented with lethargy and suction refusal. His neurological status rapidly altered in few hours and he was then immediately transferred to our institution. On admission the head circumference was above the $97^{\text {th }}$ percentile, he had already lost consciousness with anisocoric pupils and respiratory troubles. The computed tomography $(\mathrm{CT})$ scan 
showed a large tumoral mass occupying nearly the whole left hemisphere with important midline shift, intratumoral hemorrhage and wide enlargement of the cranial sutures (Figure 1A-D). Brain magnetic resonance imaging (MRI) revealed an 8 by $9 \mathrm{~cm}$ space occupying and enhancing tumor of the left temporoparietooccipital region with two areas of focal ischemia in the left frontal and right occipital regions (Figure 2A, B).
Based on these findings and the patient's clinical status, surgery was indicated in emergency and a temporoparietooccipital craniotomy was performed. Macroscopically, the tumor was gray, soft in consistency, and featured some necrotic and highly vascular areas. Progressive and careful tumor removal allowed for a subtotal resection despite very significant brain swelling. Unfortunately the patient showed no improvement post-operatively and died within two days in the intensive care unit.
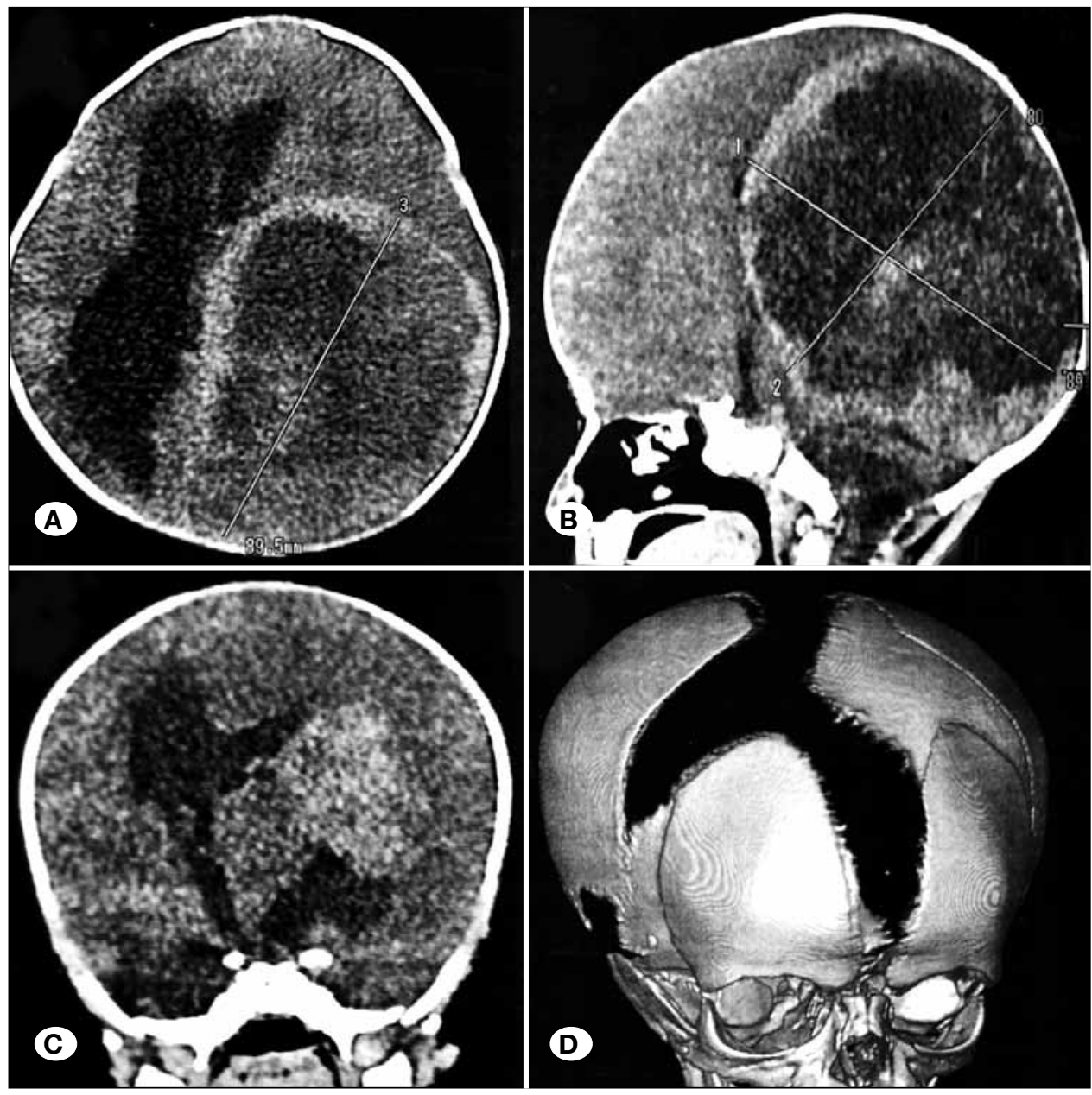

Figure 1: Axial (A), sagittal (B), coronal (C) and 3D bone reconstruction (D) CT showing a large left parietotemporooccipital mass with thick wall, central necrosis and intratumoral hemorrhage. Note the wide enlargement of the skull vault sutures (D).
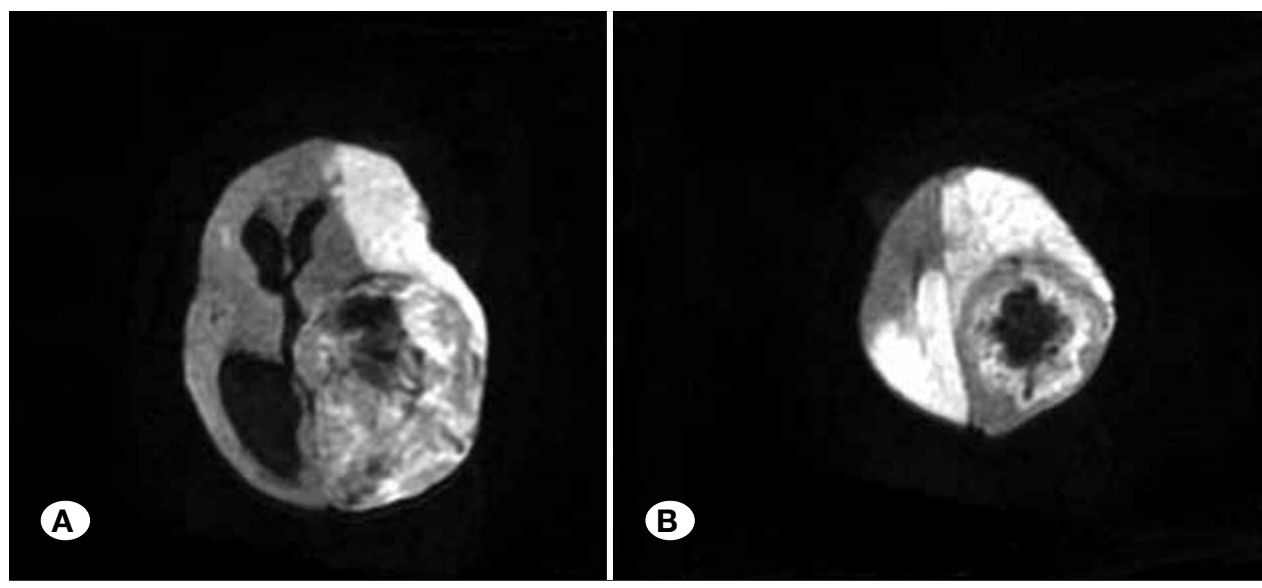

Figure 2: Axial diffusion B1000 MR images showing the tumor with two areas of focal ischemia in the left frontal $(\mathbf{A}+\mathbf{B})$ and the right parietal (B) regions. 
Prenatal ultrasound scans, supposed to be normal, were reviewed by two different radiologists and revealed a small mass of high echogenicity in the left hemisphere that appeared at the $37^{\text {th }}$ week of gestation (Figure 3).

Histological examination showed a highly cellular tumor with prominent anaplasia and large areas of pseudopalisading necrosis - a characteristic feature of high-grade gliomas. (Figure 4A-C). The Ki-67 proliferation index was $23 \%$ Immunohistochemical staining was positive for p53, epidermal growth factor receptor (EGFR) and glial fibrillary acidic protein (GFAP).

The findings are compatible with the diagnosis of congenital glioblastoma.

\section{- DISCUSSION}

First described by Holt (1) in 1917, congenital brain tumors are

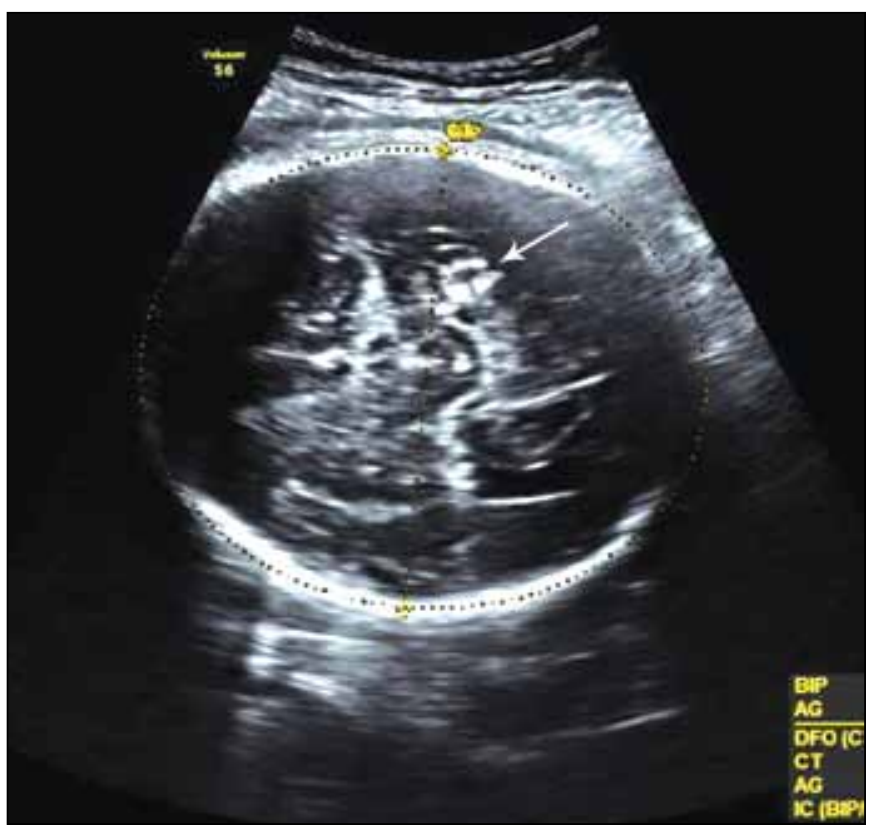

Figure 3: Thirty-seven weeks gestation transverse ultrasounds scan through fetal head showing an area of high reflectivity in the left temporoparietal region (Arrow). extremely rare and account for only 0.3 to $4 \%$ of all CNS tumors in children (4). They often arise during pregnancy and are frequently identified at autopsy (9). The Solitare and Krigman classification groups congenital brain tumors according to time at diagnosis as follow: definitely congenital (present at birth), probably congenital (detected within the 1st week of life), and possibly congenital, that is, detected within the first 2 months of life (8). Other classifications take symptoms and time of diagnosis into account and extend congenital tumor criteria to cover the first year of life. Including the present case, the total review population of congenital glioblastomas was 52 cases; only 39 of them were definitely congenital.

Most congenital GBMs occur in male infants and are more common in the supratentorial region. During pregnancy, they can present with hydrocephalus or polyhydramnios due to depressed fetal swallowing reflex. Intrauterine death and dystocia during labor have also been described. In the postnatal period: enlarged head circumference, bulging fontanel, vomiting, and seizures are the other clinical manifestations. Focal neurologic changes may not be seen in many cases because the calvaria can expand and accommodate the increasing brain volume. In the present case, physical examination was normal at birth and the baby presented in emergency with rapid neurological deterioration few hours prior to his admission.

To the best of our knowledge, such presentation has never been previously reported.

Improved imaging tools such as ultrasound have permitted increasing reliable prenatal diagnoses. Sonographically, fetal glioblastoma appear as a unilateral echogenic mass occupying most of one hemisphere. Significant midline shift and intratumoral hemorrhage is frequently seen (7).

CT of congenital glioblastoma typically reveals a lobar, mixed attenuating mass with marked annular enhancement. Calcifications are uncommon and the tumor is usually surrounded by vasogenic edema.

On both fetal and postnatal MRI, congenital glioblastomas appear hypo to isointense to the brain on T1-weighted sequences, hyperintense on T2-weighted sequences, with possible areas of hemorrhage and necrosis (6). Restricted
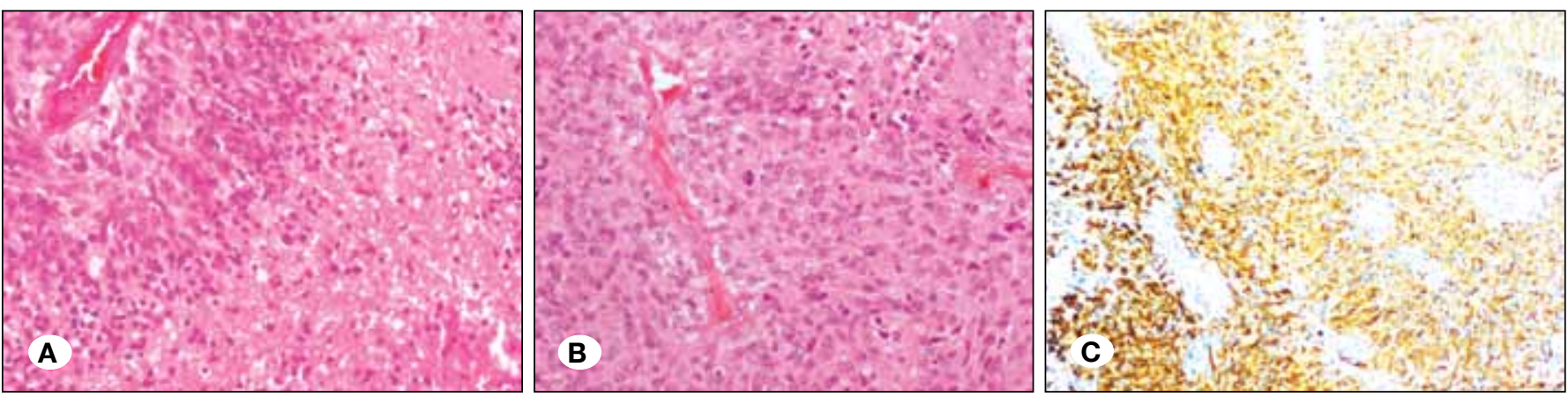

Figure 4: Photomicrographs of the tumor specimens showing in: A) Prominent anaplasia with areas of pseudo palisading (Hematoxylin \& Eosin, original magnifications x100). B) Marked nuclear polymorphism (Hematoxylin \& Eosin, original magnifications x200). C) GFAP positive staining. 
diffusion correlates with high tumor grade and differentiation of tumor from peri-tumoral edema is not always obvious (6).

Differential diagnosis of a supratentorial heterogeneous hemispheric mass in a newborn may include teratoma, choroid plexus papilloma, primitive neuroectodermal tumors and glial or mixed neuronal-glial tumors. Although some clues may help us predict the distinct histological type like the absence of coarse calcifications often seen in teratoma, differential diagnosis is still challenging.

Microscopically, congenital glioblastomas are typically densely cellular tumors, demonstrating marked pleomorphism, mitotic activity, microvascular proliferation, and necrosis. It should also be emphasized that all of these tumors arise de novo so there is no detectable lower grade precursor in these forms.

In the present case, a small area of hyper echogenicity appeared in the $37^{\text {th }}$ week ultrasound examination and was overlooked leading to a consequent diagnostic delay. Tumor characteristics on both CT and MRI were common of congenital glioblastoma and central hemorrhagic area was also found. What is remarkable, however; is the rapid enlargement of the tumor leading to a huge mass nearly occupying a whole hemisphere in a short period of time. The associated bilateral areas of ischemia, described here for the first time, compound with the huge seize of the tumor in the rapid neurological deterioration and secondarily dismal progression.

Fifty-seven of the 66 articles on congenital brain tumors published from the 1980s till 2013 focused on the tumor biology, histology or immunohistochemistry providing no clear management guidelines to address this serious condition.

Initially, the prognosis was considered universally poor and the treatment was based upon this perception. But in 2001, Winters et al. (9) in a review of 24 cases of congenital glioblastomas managed by surgery plus adjuvant chemotherapy, found an average survival rate of 32.8 months; which radically changed the prognosis of congenital glioblastomas. Chemotherapy is in fact well tolerated allowing for administration of escalating doses of drugs that cross the CNS barriers and radiation therapy is avoided because of its devastating side effects.

Being often very large and highly vascular, congenital glioblastoma surgery is also frequently challenging and risky.

Given the benefits of pre-operative embolization, Seker and Ozek (5), in 2006, successfully treated a congenital glioblastoma by preoperative embolization, radical surgery plus chemotherapy and thus outline the importance of preoperative arterial selective embolization as a preferred management policy to prevent intraoperative bleeding. More recently, Kotecha et al. (2) based on a remarkable history of two cases of congenital glioblastoma whose initial surgery was hampered by tumor hypervascularity and excessive blood loss, recommend cytoreductive chemotherapy following diagnostic biopsy in case of infants harboring large and highly vascular congenital glioblastomas. A novel approach that could increase the feasibility and safety of gross total resection at a second-look surgery but which is not feasible in all cases, like ours, requiring prompt surgical intervention.
Macy et al. (3) eloquently discussed the clinical and molecular characteristics of congenital glioblastomas. They found that congenital forms differ from pediatric and adult counterparts in the expression of a limited number of genes and that they may have a significantly better prognosis. Factors associated with poor prognosis include p53 protein accumulation/ overexpression, EGFR expression and high proliferative index $(5,9)$. All of them were present in our case and could explain such an aggressive biological behavior.

\section{CONCLUSION}

Congenital glioblastomas are very rare and can manifest in emergency with rapid neurological deterioration.

The case reported in this article outlines the importance of their early diagnosis and that they should be considered in the differential diagnosis when fetal ultrasonography or fetal $\mathrm{MRI}$ reveals a tumor, especially in the presence of intratumoral hemorrhage.

Although management guidelines are still pending, we believe that surgery plus chemotherapy should be indicated whenever possible.

\section{- REFERENCES}

1. Holt L: Gliosarcoma in an infant of several weeks resembling hydrocephalus. Am J Dis Child 39: 219-221, 1917

2. Kotecha RS, Burley K, Junckerstorff RC, Lee S, Phillips MB, Cole $\mathrm{CH}$, Gottardo NG: Chemotherapy increases amenability of surgical resection in congenital glioblastoma. Pediatr Hematol Oncol 29(6): 538-544, 2012

3. Macy ME, Birks DK, Barton VN, Chan MH, Donson AM, Kleinschmidt-Demasters BK, Bemis LT, Handler MH, Foreman NK: Clinical and molecular characteristics of congenital glioblastoma. Neuro Oncol 14(7): 931-941, 2012

4. Milano GM, Cerri C, Ferruzzi V, Capolsini I, Mastrodicasa E, Genitori L, Aversa F: Congenital glioblastoma. Pediatr Blood Cancer 53 (1): 124-126, 2009

5. Seker A, Ozek MM: Congenital glioblastoma multiforme. Case report and review of the literature. J Neurosurg 105: 473-479, 2006

6. Severino M, Schwartz ES, Thurnher MM, Rydland J, Nikas I, Rossi A: Congenital tumors of the central nervous system. Neuroradiology 52(6): 531-548, 2010

7. Shimamura N, Asano K, Ogane K, Yagihashi A, Ohkuma $\mathrm{H}$, Suzuki S: A case of definitely congenital glioblastoma manifested by intratumoral hemorrhage. Childs Nerv Syst 19(10-11):778-781, 2003

8. Solitare GB, Krigman MR: Congenital intracranial neoplasm: A case report and review of the literature. J Neuropathol Exp Neurol 23: 280-292, 1964

9. Winters JL, Wilson D, Davis DG: Congenital glioblastoma multiforme: A report of three cases and a review of the literature. J Neurol Sci 188:13-19, 2001 